\title{
Resveratrol ameliorates depressive disorder through the NETRIN1-mediated extracellular signal-regulated kinase/cAMP signal transduction pathway
}

\author{
FEIFEI WANG ${ }^{1}$, JINHUI WANG ${ }^{1}$, JINGHONG AN ${ }^{1}$, GUOMING YUAN ${ }^{1}$, XIAOLEI HAO ${ }^{1}$ and YI ZHANG ${ }^{2}$ \\ ${ }^{1}$ Clinical Laboratory; ${ }^{2}$ Department of Psychiatry and Psychology, \\ The First Hospital of Harbin, Harbin, Heilongjiang 150000, P.R. China
}

Received December 19, 2016; Accepted November 6, 2017

DOI: $10.3892 / \mathrm{mmr} .2018 .8379$

\begin{abstract}
Depressive disorder is a mental health disorder caused by the dysfunction of nerve regeneration, neuroendocrine and neurobiochemistry, which frequently results in cognitive impairments and disorder. Evidence has shown that resveratrol offers benefits for the treatment of depressive disorder. In the present study, the therapeutic effects of resveratrol were investigated and the potential mechanisms mediated by resveratrol were analyzed in hippocampal neuron cells. The anti-oxidative stress and anti-inflammatory properties of resveratrol were also examined in vitro and in vivo. The results revealed that resveratrol administration inhibited the inflammation in hippocampal neuron cells induced by ouabain. Oxidative stress in the hippocampal neuron cells was ameliorated by resveratrol treatment in vitro and in vivo. In addition, the apoptosis of hippocampal neuron cells was inhibited by the upregulation of anti-apoptotic genes, including P53, B-cell lymphoma-2 (Bcl-2) and $\mathrm{Bcl}-2$-associated death promoter, and the downregulation of the cleaved caspase- 3 and caspase- 9 . The analysis of the mechanism revealed that that resveratrol treatment suppressed the apoptosis of hippocampal neuron cells through the NETRIN1-mediated extracellular signal-regulated kinase/cAMP signal transduction pathway. The results of the in vivo assay showed that resveratrol treatment led to improvements in cognitive competence, learning memory ability and anxiety in a mouse model of depressive disorder induced by ouabain. In conclusion, these results indicated that resveratrol treatment had protective effects against oxidative stress and neuroinflammatory pathogenesis through the NETRIN1-mediated extracellular signal-regulated kinase/cAMP signal transduction pathway, suggesting that resveratrol treatment may be a potential antidepressant agent for the treatment of depressive disorder.
\end{abstract}

Correspondence to: Professor Yi Zhang, Department of Psychiatry and Psychology, The First Hospital of Harbin, 151 Diduan Street, Harbin, Heilongjiang 150000, P.R. China

E-mail: zhangyihospital@163.com

Key words: resveratrol, depressive disorder, apoptosis, oxidative stress, NETRIN1, cAMP

\section{Introduction}

Currently, depressive disorder is adding to the global burden of disease, and has been considered to be a major public health concern with a genetic cause $(1,2)$. A systematic review has showed that neurostimulatory and ablative treatment options are important in cognitive impairments in pathological processes in depressive disorder (3). Clinical investigations have found that depressive disorder can aggravate the pathological symptoms associated with cardiovascular and cerebrovascular diseases, and can cause increased disability and distress (4). Although the use of antidepressants for the treatment of depression, in addition to mindfulness-based therapies, technology-assisted self-help and minimal contact therapies, have been described in systematic reviews and meta-analysis $(5,6)$, the potential mechanisms of the pathological processes in depressive disorder have also been investigated and discussed $(7,8)$.

It has been suggested that neurobiological treatments may be efficacious for depression through structural and functional alterations to the prefrontal cortex, hippocampus and amygdala in the brain (9-11). Resveratrol is a multifunctional biological polyphenol, which has ameliorative effects on high concentrations of inflammatory factors, metabolic syndrome and neurological diseases $(12,13)$. A study by O'Donovan et al indicated that suicidal ideation is associated with elevated inflammation in patients with major depressive disorder (14). Previous reports have also demonstrated that resveratrol can effectively inhibit endothelial degeneration and prevent the production of tumor necrosis factor $\alpha$ in patients with coronary artery disease (15), and has also shown beneficial effects in patients with chronic kidney disease through the downregulation of nuclear factor erythroid 2-related factor 2 and nuclear factor $-\kappa \mathrm{B}$ in a randomized, double-blind, placebo-controlled, crossover clinical trial (16). In addition, Rodrigues et al described the potential association between oxidative stress and genetic vulnerability in major depressive disorder and Alzheimer's disease (17), and the effects of oxidative and nitrosative stress in accelerated aging and major depressive disorder have also been discussed in a previous study (18).

In the present study, the benefits of resveratrol for depressive disorder were investigated in hippocampal neuron cells 
and in a mouse model of depressive disorder. The potential mechanisms mediated by resveratrol for inflammation, apoptosis and oxidative stress were also analyzed in hippocampal neuron cells, and cognitive impairments from pathological processes were examined in the depressive disorder mouse model induced by ouabain. The overall findings suggested that resveratrol treatment inhibited the apoptosis of hippocampal neuron cells through regulation of the NETRIN1-mediated extracellular signal-regulated kinase/cAMP signal transduction pathway in the treatment of depressive disorder.

\section{Materials and methods}

Ethics statement. The present study was performed in accordance with the recommendations in the Guide for the Care and Use of Laboratory Animals of China. All operative procedures were approved by the Committee on the Ethics of Animal Experiments Defence Research at The First Hospital of Harbin (Harbin, China).

Cell culture and reagents. Resveratrol was purchased from Merck Millipore (Darmstadt, Germany) and was made up to a solution of $100 \mathrm{mg} / \mathrm{ml}$ with PBS. The hippocampal neuron cells were isolated from the experimental mice with depression as described previously (19) and cultured in Dulbecco's modified Eagle's medium supplied with $10 \%$ fetal bovine serum (Merck Millipore). The cells were cultured at $37^{\circ} \mathrm{C}$ in a humidified atmosphere of $95 \%$ air and $5 \% \mathrm{CO}_{2}$.

Western blot analysis. The hippocampal neuron cells were harvested by scraping and lysing in RIPA buffer, which followed homogenization at $4^{\circ} \mathrm{C}$ for $10 \mathrm{~min}$. Protein concentration was measured using a bicinchoninic assay protein assay kit (Thermo Fisher Scientific, Inc., Waltham, MA, USA). Protein $(10 \mu \mathrm{g})$ was analyzed using $12 \%$ SDS-PAGE assays followed by transfer onto a polyvinylidene fluoride membranes (Merck Millipore). The primary antibodies used in the immunoblotting assays were from Abcam (Cambridge, UK) as follows: IL-1 $\beta$ (1:400; cat. no. ab200478), IL-17A (1:500, cat. no. ab180904), IL-8 (1:500; cat. no. ab197016), TNF- $\alpha$ (1:500; cat. no. ab1793), superoxide dismutase (SOD; 1:500; cat. no. ab13533), glutathione (GSH; 1:500; cat. no. ab26255), neuroglobin (NEG; 1:500; cat. no. ab37258) and catalase (CAT; 1:500; cat. no. ab16731), CYC-2 (1:500, cat. no. ab188183), P53 (1:400; cat. no. ab26), Bcl-2 (1:400; cat. no. ab32124), caspase-3 (1:500; cat. no. ab217), caspase-9 (1:500; cat. no. ab52298), NETRIN1 (1:500; cat. no. ab39370), neuregulin 3 (NRG3; 1:500; cat. no. ab109256), cAMP (1:500; cat. no. ab26322) and $\beta$-actin (1:1,000, cat. no. ab5694). Horseradish peroxidase-conjugated anti-rabbit IgG antibodies (PV-6001, OriGene Technologies, Inc., Beijing, China) were used at a 1:5,000 dilution and detected using western blotting luminol reagent (Thermo Fisher Scientific, Inc.). Band density was analyzed using Quantity One software (version 4.62; Bio-Rad Laboratories, Inc.).

Small interfering RNA (siRNA) transfection. The hippocampal neuron cells were cultured to $85 \%$ confluence and transfected with siRNA targeting NETRIN1 (si-NETRIN1) or scrambled si-vector using Lipofectamine ${ }^{\mathrm{TM}}$ RNAi MAX (Invitrogen;
Thermo Fisher Scientific, Inc.) according to the manufacturer's protocol. The siRNA targeting NETRIN1 (sense, 5'-CUU AGCUAGCCAUAUUCCUUCCA-3'; antisense, 5'-AUAUCG UGUAAC UCGUCUAGAU-3') and scrambled siRNA (sense, 5'-AUCACGUACGCGGAAUACUUCGA-3'; antisense, 5'-UCGAAGUAUUCCGCGUACGUGAU-3') were obtained from GenePharma (Shanghai, China).

Terminal deoxynucleotidyl transferase (TdT)-mediated dUTP nick-end labeling (TUNEL) assay. To analyze the apoptosis of hippocampal neuron cells in the experimental mice following treatment with resveratrol $(10 \mathrm{mg} / \mathrm{kg})$ or the same dose of PBS, a TUNEL assay (Biotool, Houston, TX, USA) was used to detect TUNEL-positive cells. The procedures were performed as described in a previous study (20). Finally, images of the hippocampal neuron cells were captured with a ZEISS LSM 510 confocal microscope (Zeiss AG, Oberkochen, Germany) at $488 \mathrm{~nm}$.

Animal experiments. Female J20 mice (6-8 weeks old, 25-32 g body weight) were purchased from Jackson Laboratory (Ben Harbor, ME, USA) and bred at Tongji Hospital, Huazhong University of Science and Technology (Wuhan, China). All animals were housed at $23 \pm 1^{\circ} \mathrm{C}$ and a relative humidity of $50 \pm 5 \%$, with a 12-h light/dark cycle and free access to food and water. The experimental mice were used to establish depressive disorder model mice via intravenously injecting $5 \mathrm{ml}$ ouabain $\left(10^{-3} \mathrm{M}\right)$ once a week for 7 weeks consecutively. The mice were separated into two groups ( $n=20$ in each group). The experimental mice were treated with a single daily oral dose of resveratrol $(10 \mathrm{mg} / \mathrm{kg})$ for 10 weeks or with the same dose of PBS as a control.

Assessment of depressive behavior. The efficacy of simvastatin treatment for depressive disorder was determined using the open field test, elevated plus maze, Barnes maze performance, object recognition, and passive avoidance experiments. These experiments were performed according to methods described in previous reports $(21,22)$.

Enzyme-linked immunosorbent assay (ELISA). The serum levels of IL-8 (cat. no. KHC0081; Thermo Fisher Scientific, Inc.), IL-1 $\beta$ (cat. no. MBS700340; Thermo Fisher Scientific, Inc.), IL-17A (cat. no. 88-7876-88; Thermo Fisher Scientific, Inc.), H3R (cat no. orb154346; Biorbyt, Cambridge, UK) and TNF- $\alpha$ (cat. no. KHC3014; Thermo Fisher Scientific, Inc.), were detected in mice with depressive disorder prior to and following treatment with resveratrol using ELISA kits according to the manufacturer's protocol. Finally, the serum concentration levels of IL-8, IL-1 $\beta$, IL-17A and TNF- $\alpha$ were measured using an enzyme microplate reader at $450 \mathrm{~nm}$.

Statistical analysis. The results are presented as the mean \pm standard error of the mean. Significance was established with SPSS 19.0 (IBM Corp., Armonk, NY, USA) and GraphPad Prism 6 software (GraphPad Software, Inc., La Jolla, CA, USA). A Kaplan-Meier plot, log-rank test, multi-variant Cox regression analysis, Pearson's correlation coefficient and a two-tailed Student's t-test were used. $\mathrm{P}<0.05$ was considered to indicate a statistically significant difference. 
A

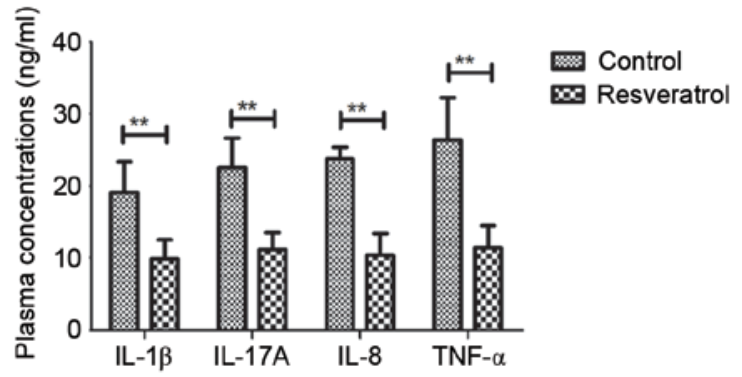

B
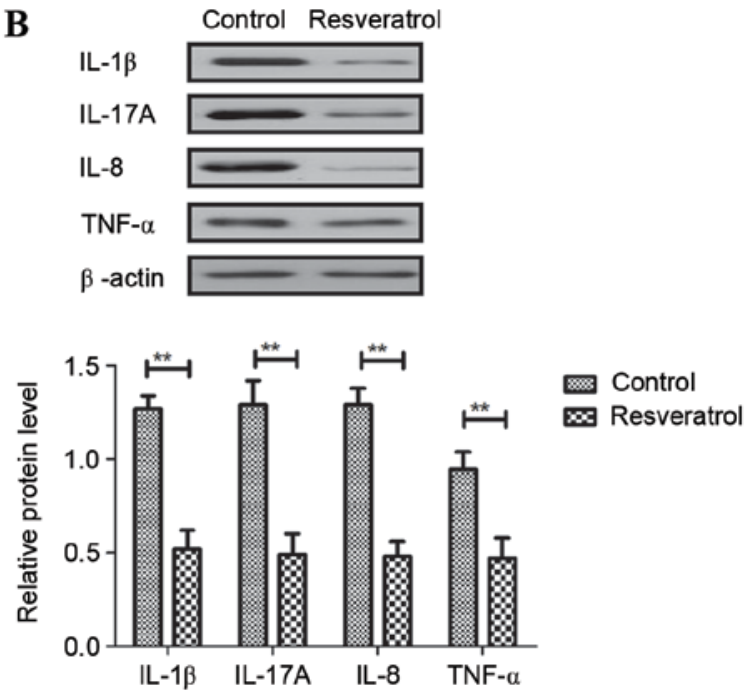

Figure 1. Resveratrol inhibits inflammatory cytokines in serum and hippocampal neuron cells of experimental mice. (A) Plasma concentrations of IL-1 $\beta$, IL-17A, IL- 8 and TNF- $\alpha$ in a mouse model of depressive disorder. (B) Protein expression levels of IL-1 $\beta$, IL-17A, IL- 8 and TNF- $\alpha$ in hippocampal neuron cells of experimental mice. ${ }^{* *} \mathrm{P}<0.01$. IL, interleukin; TNF- $\alpha$, tumor necrosis factor- $\alpha$.

\section{Results}

Resveratrol treatment inhibits inflammatory cytokines in serum and hippocampal neuron cells in experimental mice. Inflammatory cytokines are crucial in the progression of depressive disorder. Therefore, the present study investigated the expression levels of inflammatory cytokines. The results showed that resveratrol treatment downregulated the plasma concentrations of IL-1 $\beta$, IL-17A, IL-8 and TNF- $\alpha$ (Fig. 1A). The expression levels of these inflammatory cytokines were also decreased by resveratrol in hippocampal neuron cells, determined using western blot analysis (Fig. 1B). These results suggested that resveratrol treatment inhibited inflammatory cytokines in the mouse depressive disorder model.

Resveratrol decreases oxidative stress in hippocampal neuron cells in experimental mice. Oxidative stress parameters are important prognostic indicators following therapy for patients with depressive disorder (23). The data obtained in the present study showed that the production of SOD and GSH were decreased by resveratrol in the hippocampal neuron cells (Fig. 2A). As shown in Fig. 2B, the expression levels of NEG and CAT were also downregulated by resveratrol in the hippocampal neuron cells. The immunohistochemistry showed that cyclooxygenase- 2 was decreased by resveratrol in the hippocampal neuron cells (Fig. 2C). ELISA results also revealed that the serum levels of H3R were significantly lower following treatment with resveratrol (Fig. 2D). These results suggested that resveratrol treatment decreased oxidative stress in the hippocampal neuron cells of the experimental mice.

Resveratrol suppresses the apoptosis of hippocampal neuron cells in a mouse model of depressive disorder. The present study analyzed the apoptosis of hippocampal neuron cells in a mouse model of depressive disorder following resveratrol treatment. As shown in Fig. 3A, the apoptotic rate was suppressed in the resveratrol-treated hippocampal neuron cells. The protein expression levels of anti-apoptotic P53 and Bcl-2 were upregulated by resveratrol treatment in the hippocampal neuron cells (Fig. 3B). Resveratrol treatment downregulated the protein expression levels of pro-apoptotic cleaved caspase-3 and caspase-9 in the hippocampal neuron cells (Fig. 3C). The viability of the hippocampal neuron cells was also improved by resveratrol treatment (Fig. 3D). These results suggested that resveratrol suppressed the apoptosis of hippocampal neuron cells in the mouse model of depressive disorder.

Resveratrol regulates apoptosis of hippocampal neuron cells through the NETRIN1-mediated extracellular signal-regulated kinase/cAMP signal transduction pathway. The potential mechanism underlying the resveratrol-regulated apoptosis of hippocampal neuron cells was examined. As shown in Fig. 4A, the expression levels of NETRIN1 and NRG3 were increased in the hippocampal neuron cells treated with resveratrol. The administration of resveratrol inhibited the activity and expression of cAMP in the hippocampal neuron cells (Fig. 4B and C). The knockdown of NETRIN1 (siNETRIN1) eliminated the resveratrol-induced inhibition (siNET-RS) of the activity and expression of cAMP in the hippocampal neuron cells (Fig. 4D and E). Apoptosis was also increased in the NETRIN1-knockdown hippocampal neuron cells following treatment with resveratrol (Fig. 4F), and the effects on the gene expression levels of P53, Bcl-2, cleaved caspase- 3 and caspase- 9 were also reversed in the resveratrol-treated hippocampal neuron cells (Fig. 4G and $\mathrm{H}$ ). These results suggested that resveratrol regulated the apoptosis of hippocampal neuron cells through the NETRIN1-mediated extracellular signal-regulated kinase/cAMP signal transduction pathway.

Resveratrol administration ameliorates depressive behaviors in mice with depressive disorder induced by ouabain. The present study further analyzed the therapeutic efficacy of resveratrol on the mouse model induced by ouabain. As shown in Fig. 5A, resveratrol treatment relieved anxiety, which was determined by the time spent in the center. The resveratrol-treated mice 
A
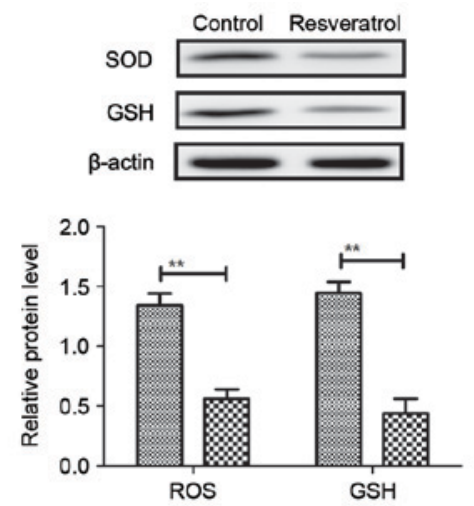

C

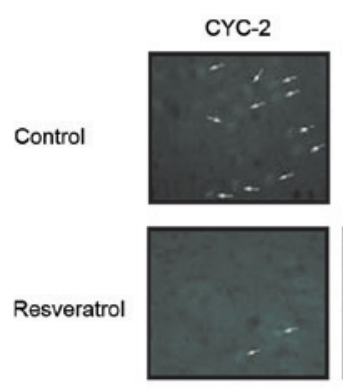

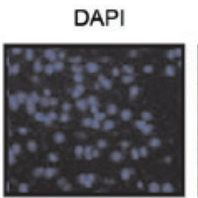

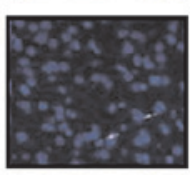

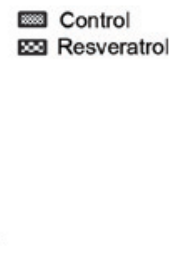

Merge
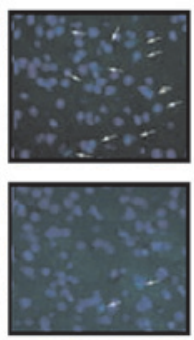

B
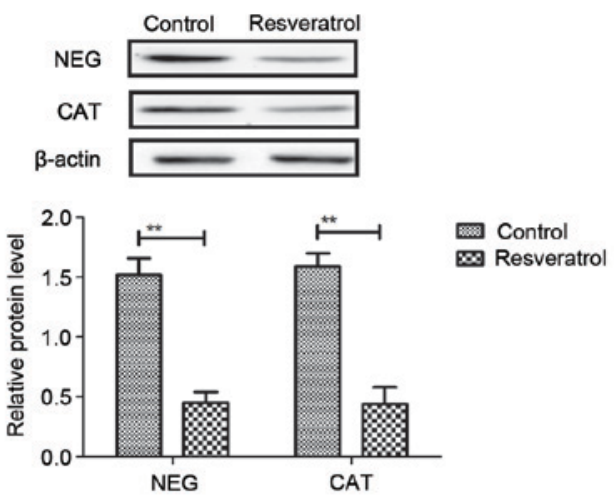

D

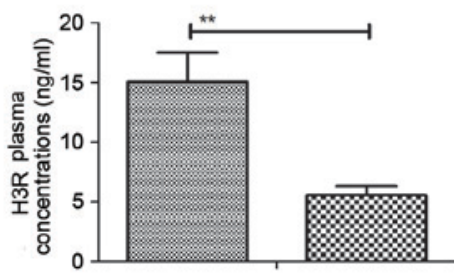

Control

Resveratrol

Figure 2. Resveratrol decreases oxidative stress in hippocampal neuron cells of experimental mice. (A) Resveratrol decreased the production of SOD and GSH in hippocampal neuron cells. (B) Effects of resveratrol on NEG and CAT in hippocampal neuron cells. (C) Expression levels of CYC-2 in hippocampal neuron cells, determined using immunohistochemistry. Magnification, x40. Arrows indicate CYC-2 positive cells. (D) Effects of resveratrol on serum levels of H3R in hippocampal neuron cells. ** $\mathrm{P}<0.01$. SOD, superoxide dismutase; GSH, glu tathione; NEG, neuroglobin; CAT, catalase; CYC-2, cyclooxygenase-2.

A

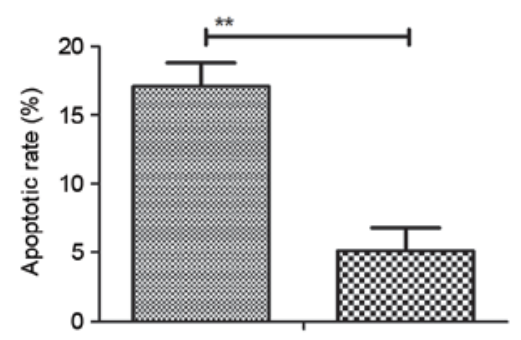

C
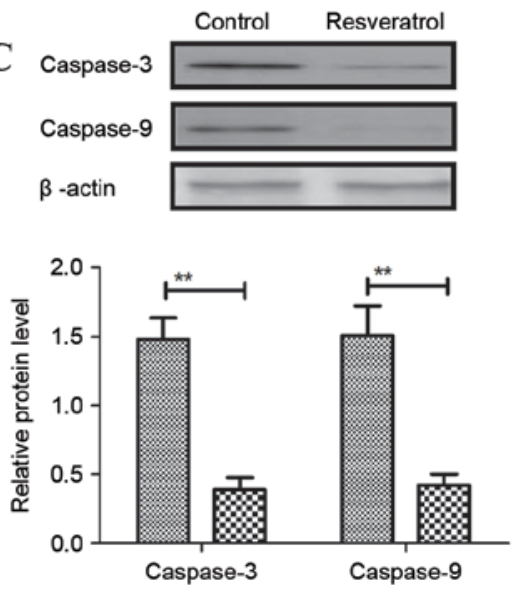

B

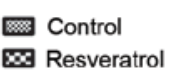

Control

$\infty$ Resveratrol

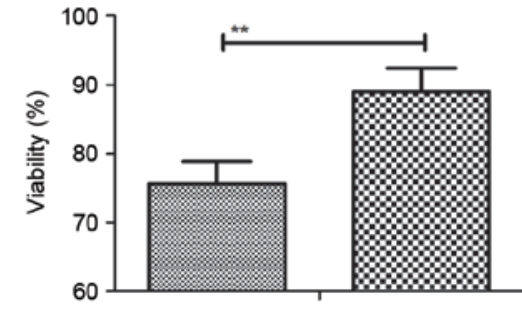

D
Control

$\infty$ Resveratrol

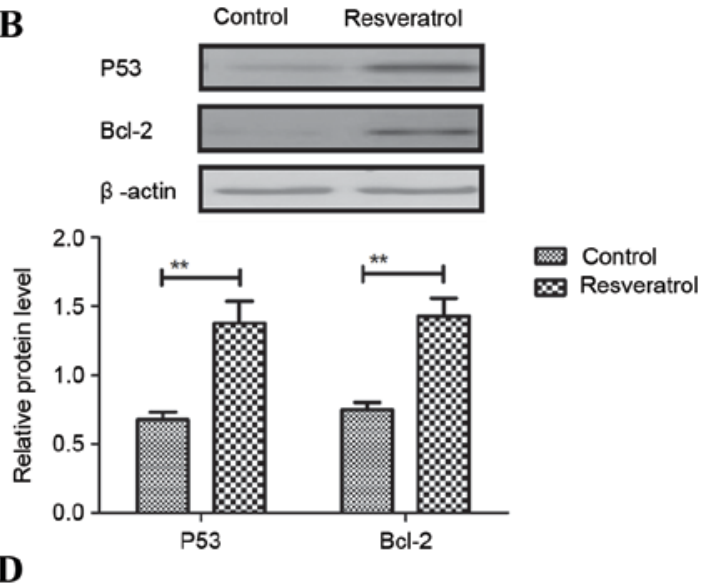

Control

$\infty 8$ Resveratrol

Figure 3. Resveratrol suppresses the apoptosis of hippocampal neuron cells in a mouse model of depressive disorder. (A) Resveratrol inhibited the apoptosis of hippocampal neuron cells in mice with depressive disorder. (B) Protein expression levels of anti-apoptotic P53 and Bcl-2 in hippocampal neuron cells following treatment with resveratrol. (C) Protein expression levels of pro-apoptostic cleaved caspase-3 and caspase-9 in hippocampal neuron cells following treatment with resveratrol. (D) Resveratrol improved the viability of hippocampal neuron cells. ${ }^{* * *} \mathrm{P}<0.01$. Bcl-2, B-cell lymphoma 2. 
$\mathbf{A}$
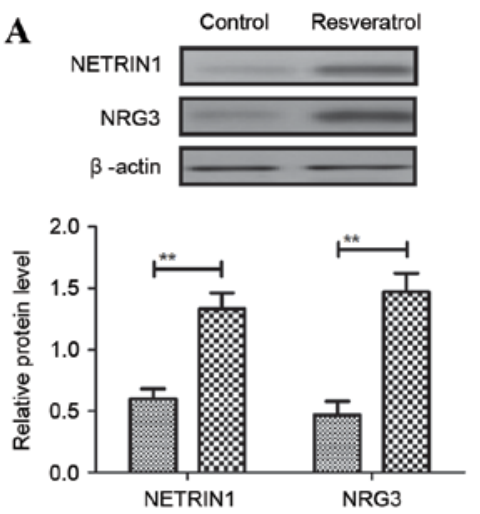

c
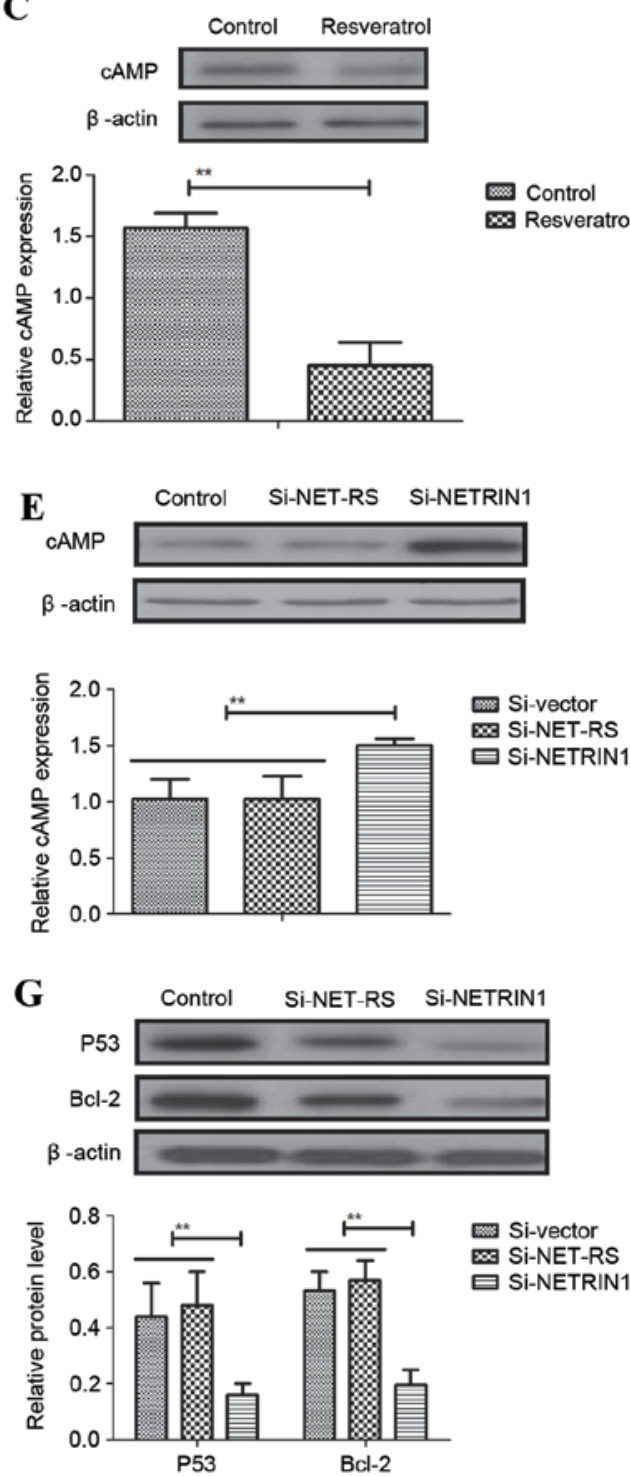

B

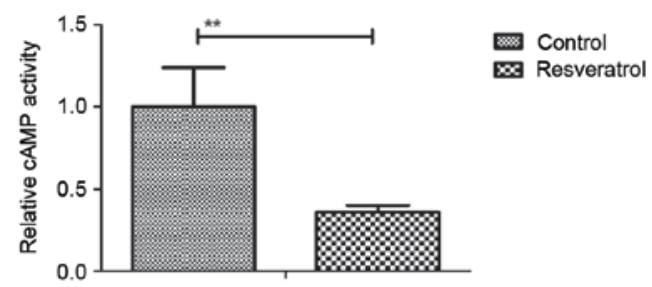

D

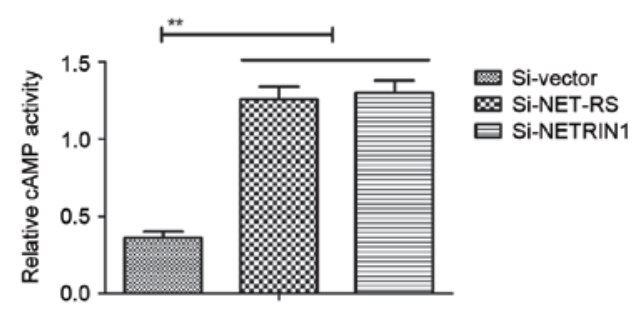

$\mathbf{F}$

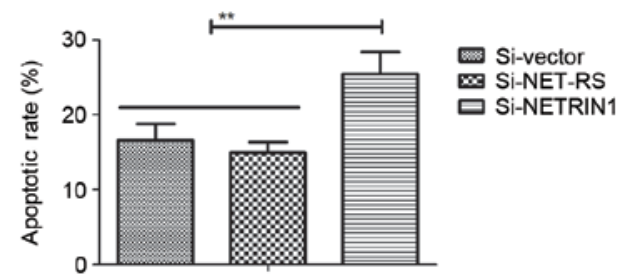

H
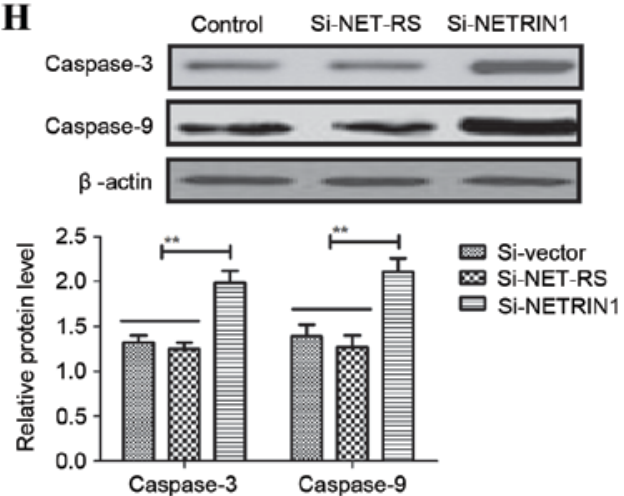

Figure 4. Resveratrol inhibits the apoptosis of hippocampal neuron cells through the NETRIN1-mediated cAMP signal transduction pathway. (A) Resveratrol increased the expression levels of NETRIN1 and NRG3 in hippocampal neuron cells. Resveratrol administration inhibited the (B) activity and (C) expression of cAMP in hippocampal neuron cells. Knockdown of NETRIN1 increased the (D) activity and (E) expression of cAMP in hippocampal neuron cells. (F) NETRIN1 knockdown reversed the resveratrol-induced downregulated apoptosis of hippocampal neuron cells. NETRIN1 knockdown reversed the resveratrol-regulated effects on expression levels of $(\mathrm{G})$ anti-apoptotic and $(\mathrm{H})$ pro-apoptotic genes in hippocampal neuron cells. ${ }^{* * *} \mathrm{P}<0.01$. Si, small interfering RNA; NRG3, neuregulin 3; Bcl-2, B-cell lymphoma 2.

showed increased movement and took less time to recognize a novel object, compared with the control mice (Fig. 5B and C). In addition, the body weights of the mice were improved following resveratrol treatment, compared with the control group (Fig. 5D). Collectively, these results suggested that resveratrol treatment ameliorated depressive behaviors. 

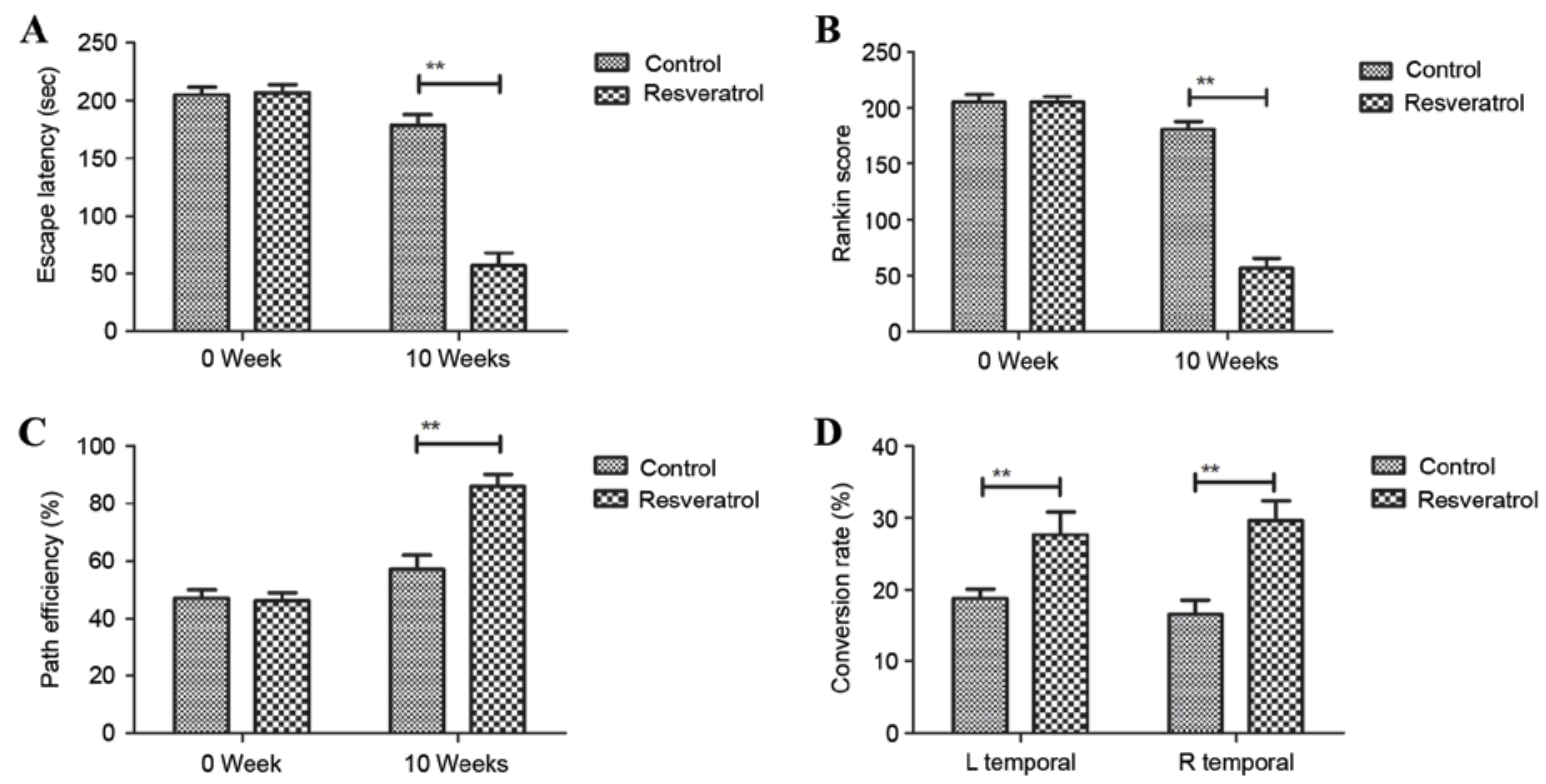

Figure 5. Resveratrol treatment improves depressive behaviors in a mouse model of depressive disorder induced by ouabain. (A) Resveratrol treatment relieved anxiety, determined by the duration the mouse spent in the center. Resveratrol treatment improved the (B) distance moved and (C) reduced the time taken to recognize a novel object in the mouse model of depressive disorder induced by ouabain. (D) Resveratrol treatment improved the body weights of mice in the model of depressive disorder induced by ouabain. ${ }^{* *} \mathrm{P}<0.01$.

\section{Discussion}

Depressive disorder presents with an increasing trend of a number of biological psychological factors and frequently co-occurs with other psychiatric problems $(24,25)$. Evidence indicates that inflammation and apoptosis contribute to the initiation and development of depressive disorder, and are regarded as prognostic indicators in the treatment of depression $(26,27)$. Previously, it has been shown that resveratrol can ameliorate depressive-like behavior in repeated corticosterone-induced depression in mice (28). However, the potential mechanisms underlying the resveratrol-mediated beneficial effects in hippocampal neuron cells remain to be fully elucidated. The present study investigated the therapeutic effects of resveratrol in mice with depressive disorder induced by ouabain, and examined the molecular mechanism mediated by resveratrol in hippocampal neuron cells. The findings suggested that resveratrol administration ameliorated the depressive behaviors in the mice with depressive disorder induced by ouabain. This occurred by the inhibition of hippocampal neuron cell apoptosis through the NETRIN1-mediated extracellular signal-regulated kinase/cAMP signal transduction pathway.

Inflammatory cytokines and depression are closely associated as inflammation induces the symptoms of depression and depressed mood, and favors stress, indicating the ability of inflammation to induce symptoms of depression (29). Kohler et al showed that anti-inflammatory treatments are efficient for patients with depression, and attributed to learning and memory ability $(30,31)$. Resveratrol has been shown to attenuate inflammation via the modulation of intracellular GSH homeostasis in the processes of atherosclerosis and ischemic brain injury in aged female mice $(32,33)$. In the present study, the results showed that resveratrol treatment inhibited inflammatory cytokines in the hippocampal neuron cells isolated from mice with depressive disorder.
The oxidative stress and nitrosative stress pathways in major depression may contribute to the degenerative processes in the hippocampal region (34). Oxidative stress and inflammatory markers also have been associated with depression and nicotine dependence (35). A study by Mathieu et al indicated that resveratrol attenuated oxidative stress in mitochondrial complex I deficiency through regulation of the sirtuin 3 signaling pathway (36). In the present study, it was observed that resveratrol treatment attenuated oxidative stress in hippocampal neuron cells, which may have contributed to the recovery in depressive disorder.

The apoptosis of hippocampal neuron cells impairs cognition and increases anxiety in patients with depressive disorder $(37,38)$. It has been demonstrated that resveratrol treatment can inhibit testicular dysfunction and apoptosis via involvement of the p38 mitogen-activated protein kinase/activating transcription factor $2 /$ inducible nitric oxide synthase signaling pathway (39). Resveratrol has also been reported to improve hippocampal atrophy in mice with chronic fatigue by enhancing neurogenesis and inhibiting the apoptosis of granular cells (40). In the present study, the results of the analyses suggested that resveratrol treatment inhibited the apoptosis of hippocampal neuron cells. Examination of the potential mechanism indicated that resveratrol regulated the apoptosis of hippocampal neuron cells through the NETRIN1-mediated extracellular signal-regulated kinase/cAMP signal transduction pathway, which is constant with previous reports $(41,42)$.

In conclusion, the results of the present study demonstrated that inflammation and oxidative stress are involved in the pathological processes of depressive disorder. Notably, these findings demonstrated the importance of anti-apoptotic effects on hippocampal neuron cells for protecting against cognitive impairments in the mouse model of depression induced by ouabain. The results also suggested that inhibition of the NETRIN1-mediated extracellular signal-regulated kinase/cAMP signal transduction 
pathway mediated by resveratrol contributed to cognitive repair and decreased anxiety in the experimental mice.

\section{Acknowledgements}

This study was supported by the Talent Introduction Project of The First Hospital of Harbin (grant no. 2013SYYRCYJ01-3).

\section{References}

1. Kato M and Serretti A: Review and meta-analysis of antidepressant pharmacogenetic findings in major depressive disorder. Mo Psychiatry 15: 473-500, 2010.

2. Whale R, Terao T, Cowen P, Freemantle N and Geddes J: Pindolol augmentation of serotonin reuptake inhibitors for the treatment of depressive disorder: a systematic review. J Psychopharmacol 24 513-520, 2010.

3. Andrade P, Noblesse LH, Temel Y, Ackermans L, Lim LW, Steinbusch HWM and Vandewalle VV: Neurostimulatory and ablative treatment options in major depressive disorder: a systematic review. Acta Neurochir (Wien) 152: 565-577, 2010.

4. Carnevale T: An integrative review of adolescent depression screening instruments: applicability for use by school nurses. J Child Adolesc Psychiatr Nurs 24: 51-57, 2011.

5. Rayner L, Price A, Evans A, Valsraj K, Hotopf $M$ and Higginson IJ: Antidepressants for the treatment of depression in palliative care: Systematic review and meta-analysis. Palliat Med 25: 36-51,2011.

6. Evans S: Review: Mindfulness-based therapies effective for anxiety and depression. Evid Based Ment Health 13: 116, 2010

7. Garnock-Jones KP and McCormack PL: Escitalopram: A review of its use in the management of major depressive disorder in adults. CNS drugs 24: 769-796, 2010.

8. Croxtall JD and Scott LJ: Olanzapine/fluoxetine: A review of its use in patients with treatment-resistant major depressive disorder. CNS drugs 24: 245-262, 2010.

9. Sharpley CF: A review of the neurobiological effects of psychotherapy for depression. Psychotherapy (Chic) 47: 603-615, 2010.

10. Foland-Ross LC, Hardin MG and Gotlib IH: Neurobiological markers of familial risk for depression. Curr Top Behav Neurosci 14: 181-206, 2013.

11. Sorokina ND, Selitskii GV and Kositsyn NS: Neurobiological approach to brain functional asymmetry in depression. Usp Fiziol Nauk 36: 84-93, 2005 (In Russian).

12. Chen S, Zhao X, Ran L, Wan J, Wang X, Qin Y, Shu F, Gao Y, Yuan L, Zhang Q and Mi M: Resveratrol improves insulin resistance, glucose and lipid metabolism in patients with non-alcoholic fatty liver disease: A randomized controlled trial. Dig Liver Dis 47: 226-232, 2015.

13. Ma T, Tan MS, Yu JT and Tan L: Resveratrol as a therapeutic agent for Alzheimer's disease. Biomed Res Int 2014: 350516, 2014.

14. O'Donovan A, Rush G, Hoatam G, Hughes BM, McCrohan A, Kelleher C, O'Farrelly C and Malone KM: Suicidal ideation is associated with elevated inflammation in patients with major depressive disorder. Depress Anxiety 30: 307-314, 2013.

15. Chekalina NI, Kazakov YM, Mamontova TV, Vesnina LE and Kaidashev IP: Resveratrol more effectively than quercetin reduces endothelium degeneration and level of necrosis factor alpha in patients with coronary artery disease. Wiad Lek 69: 475-479, 2016.

16. Saldanha JF, Leal VO, Rizzetto F, Grimmer GH, Ribeiro-Alves M, Daleprane JB, Carraro-Eduardo JC and Mafra D: Effects of resveratrol supplementation in Nrf2 and NF-kappaB expressions in nondialyzed chronic kidney disease patients: A randomized, double-blind, placebo-controlled, crossover clinical trial. J Ren Nutr 26: 401-406, 2016.

17. Rodrigues R, Petersen RB and Perry G: Parallels between major depressive disorder and Alzheimer's disease: Role of oxidative stress and genetic vulnerability. Cell Mol Neurobiol 34: 925-949, 2014.

18. Maurya PK, Noto C, Rizzo LB, Rios AC, Nunes SO, Barbosa DS, Sethi S, Zeni M, Mansur RB, Maes M and Brietzke E: The role of oxidative and nitrosative stress in accelerated aging and major depressive disorder. Prog Neuropsychopharmacol Biol Psychiatry 65: 134-144, 2016.
19. Park JS, Kim S, Han DK, Lee JY and Ghil SH: Isolation of neural precursor cells from skeletal muscle tissues and their differentiation into neuron-like cells. Exp Mol Med 39: 483-490, 2007.

20. Naganuma Y,Ichii O, Otsuka S, Hashimoto Y and Kon Y: Analysis of TdT-mediated dUTP nick end labeling (TUNEL)-positive cells associated with cardiac myogenesis in mouse embryo. J Vet Med Sci 75: 283-290, 2013.

21. Castillo F, Francis L, Wylie-Rosett J and Isasi CR: Depressive symptoms are associated with excess weight and unhealthier lifestyle behaviors in urban adolescents. Child Obes 10: 400-407, 2014.

22. Zatzick D, Russo J, Lord SP, Varley C, Wang J, Berliner L, Jurkovich G, Whiteside LK, O'Connor S and Rivara FP: Collaborative care intervention targeting violence risk behaviors, substance use, and posttraumatic stress and depressive symptoms in injured adolescents: A randomized clinical trial. JAMA pediatrics 168: 532-539, 2014.

23. Galecki P, Szemraj J, Bienkiewicz M, Zboralski K and Galecka E: Oxidative stress parameters after combined fluoxetine and acetylsalicylic acid therapy in depressive patients. Hum Psychopharmacol 24: 277-286, 2009.

24. Wesnes KA, Gommoll C, Chen C, Sambunaris A, McIntyre RS and Harvey PD: Effects of levomilnacipran extended-release on major depressive disorder patients with cognitive impairments: Post-hoc analysis of a phase III study. Int Clin Psychopharmacol 32: 72-79, 2017.

25. Riepe MW, Gritzmann P and Brieden A: Preferences of psychiatric practitioners for core symptoms of major depressive disorder: A hidden conjoint analysis. Int J Methods Psychiatr Res 26: 2017.

26. Rapaport MH, Nierenberg AA, Schettler PJ, Kinkead B, Cardoos A, Walker R and Mischoulon D: Inflammation as a predictive biomarker for response to omega-3 fatty acids in major depressive disorder: A proof-of-concept study. Mol Psychiatry 21: 71-79, 2016.

27. Miguel-Hidalgo JJ, Whittom A, Villarreal A, Soni M, Meshram A, Pickett JC, Rajkowska G and Stockmeier CA: Apoptosis-related proteins and proliferation markers in the orbitofrontal cortex in major depressive disorder. J Affect Disord 158: 62-70, 2014

28. Ali SH, Madhana RM, K V A, Kasala ER, Bodduluru LN, Pitta S, Mahareddy JR and Lahkar M: Resveratrol ameliorates depressive-like behavior in repeated corticosterone-induced depression in mice. Steroids 101: 37-42, 2015.

29. Remus JL and Dantzer R: Inflammation models of depression in rodents: Relevance to psychotropic drug discovery. Int $\mathbf{J}$ Neuropsychopharmacol 19, 2016.

30. Kandasamy R, Calsbeek JJ and Morgan MM: Analysis of inflammation-induced depression of home cage wheel running in rats reveals the difference between opioid antinociception and restoration of function. Behav Brain Res 317: 502-507, 2017.

31. Kohler O, Krogh J, Mors O and Benros ME: Inflammation in depression and the potential for anti-inflammatory treatment. Curr Neuropharmacol 14: 732-742, 2016.

32. Jeong SI, Shin JA, Cho S, Kim HW, Lee JY, Kang JL and Park EM: Resveratrol attenuates peripheral and brain inflammation and reduces ischemic brain injury in aged female mice. Neurobiol Aging 44: 74-84, 2016.

33. Vasamsetti SB, Karnewar S, Gopoju R, Gollavilli PN, Narra SR, Kumar JM and Kotamraju S: Resveratrol attenuates monocyte-to-macrophage differentiation and associated inflammation via modulation of intracellular GSH homeostasis: Relevance in atherosclerosis. Free Radic Biol Med 96: 392-405, 2016.

34. Maes M, Galecki P, Chang YS and Berk M: A review on the oxidative and nitrosative stress (O\&NS) pathways in major depression and their possible contribution to the (neuro)degenerative processes in that illness. Prog Neuropsychopharmacol Biol Psychiatry 35: 676-692, 2011.

35. Vargas HO, Nunes SO, de Castro MR, Vargas MM, Barbosa DS, Bortolasci CC, Venugopal K, Dodd S and Berk M: Oxidative stress and inflammatory markers are associated with depression and nicotine dependence. Neurosci Lett 544: 136-140, 2013.

36. Mathieu L, Costa AL, Le Bachelier C, Slama A, Lebre AS, Taylor RW, Bastin J and Djouadi F: Resveratrol attenuates oxidative stress in mitochondrial Complex I deficiency: Involvement of SIRT3. Free Radic Biol Med 96: 190-198, 2016.

37. Zepeda N, Solano S, Copitin N, Chávez JL, Fernández AM, García F, Tato P and Molinari JL: Apoptosis of mouse hippocampal cells induced by Taenia crassiceps metacestode factor. J Helminthol 91: 215-221, 2017. 
38. Hsiao CJ, Lin CL, Lin TY, Wang SE and Wu CH: Imidacloprid toxicity impairs spatial memory of echolocation bats through neural apoptosis in hippocampal CA1 and medial entorhinal cortex areas. Neuroreport 27: 462-468, 2016.

39. Banerjee B, Nandi P, Chakraborty S, Raha S, Sen PC and Jana K: Resveratrol ameliorates benzo(a)pyrene-induced testicular dysfunction and apoptosis: Involvement of p38 MAPK/ATF2/iNOS signaling. J Nutr Biochem 34: 17-29, 2016.

40. Moriya J, Chen R, Yamakawa J, Sasaki K, Ishigaki Y and Takahashi T: Resveratrol improves hippocampal atrophy in chronic fatigue mice by enhancing neurogenesis and inhibiting apoptosis of granular cells. Biol Pharm Bull 34: 354-359, 2011.
41. Naviglio S, Di Gesto D, Romano M, Sorrentino A, Illiano F, Sorvillo L, Abbruzzese A, Marra M, Caraglia M, Chiosi E, et al: Leptin enhances growth inhibition by cAMP elevating agents through apoptosis of MDA-MB-231 breast cancer cells. Cancer Biol Ther 8: 1183-1190, 2009.

42. Naderi EH, Findley HW, Ruud E, Blomhoff HK and Naderi S: Activation of cAMP signaling inhibits DNA damage-induced apoptosis in BCP-ALL cells through abrogation of p53 accumulation. Blood 114: 608-618, 2009. 